\title{
The RHIC Tune Measurement System
}

\author{
P. Cameron, R. Connolly, J. Cupolo, A. Drees, W. Ryan, T. Shea, \\ R. Sikora, D. Trbojevic, and N. Tsoupas \\ Brookhaven National Lab, Upton, NY 11973
}

\begin{abstract}
Each of the four fractional betatron tunes of RHIC (two planes, two rings) is measured with a transverse beam kicker and dedicated beam position monitor (BPM). A 1$5 \mathrm{kV}$ pulse is generated by a fast FET switch, passed through a stripline kicker, attenuated, and returned to the instrumentation control room (ICR) for monitoring. When pulsed at $3 \mathrm{kV}$, the four-meter long kicker gives a gold beam at injection $(\gamma=12.6)$ an angular kick of $11 \mu \mathrm{rad}$ resulting in a betatron amplitude of $0.8 \mathrm{~mm}$ at the BPM $\left(\sigma_{\text {beam }}=4.3 \mathrm{~mm}\right)$. A single bunch is kicked a few times and then measured for several hundred turns. A fast Fourier transform (FFT) of the position data gives the tune.
\end{abstract}

\section{INTRODUCTION}

The Relativistic Heavy Ion Collider (RHIC) at Brookhaven National Lab consists of two synchrotrons which intersect at six points around the $3.8 \mathrm{~km}$ circumference [1]. Ion beams from protons to fullystripped gold will be accelerated and stored. Both rings are designed to have horizontal tunes of 28.19 and vertical tunes of 29.18. Each fractional tune will be measured by exciting a betatron oscillation in a single bunch with a transverse beam kicker and measuring the transverse position on subsequent turns with a BPM [2].

Each ring has two kicker modules with four $2 \mathrm{~m}$-long striplines to allow both horizontal and vertical kicks. Two kickers are installed in a ring so one can be used with pulsed power for kicks and the other can be driven proportionally with rf amplifiers for transverse damping. For early operation of RHIC only pulsed power will be used and the two kickers are connected in series to provide $4 \mathrm{~m}$ of stripline kicker. Additionally only one stripline in each plane will be powered and the opposing stripline will be terminated on both ends.

In this paper we describe the kicker and the BPM. We describe the timing which allows the selection of the same bunch for the kicker and BPM. Finally results are given of simulations done to determine the measurement accuracy possible considering the RHIC tune spread and $\mathrm{BPM}$ resolution.

\section{KICKER AND BPM}

Figure 1 shows one section of kicker in the assembly area set up for electrical measurements. Four 2m-long, stainless steel striplines are mounted on ceramic standoffs spaced $1 \mathrm{~m}$ apart. Each subtends an angle of $70^{\circ}$ and the aperture is $7 \mathrm{~cm}$. Electrical connectors are type $\mathrm{HN}$ feedthroughs. The assembly is designed to give $50 \Omega$ impedance when opposing lines are driven in the difference mode. With three lines terminated the impedance of a single line is $55 \Omega$.

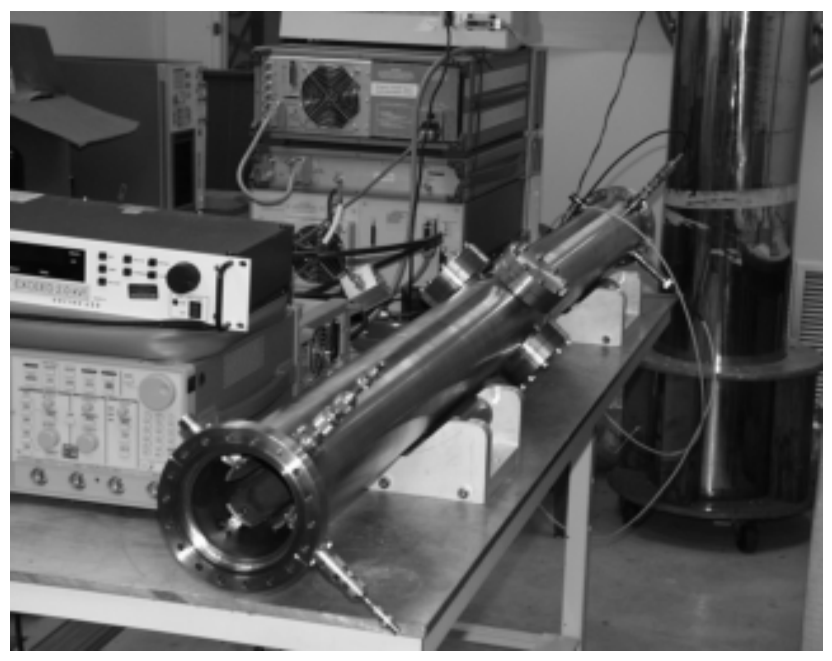

Figure 1. One section of kicker in assembly area.

One channel of the kicker system is shown in fig. 2 . The kick pulses are generated by fast FET switches made by Behlke [3]. Pulse current is supplied by capacitors on the switch input which are charged by a $5 \mathrm{kV}$ power supply. The switches for all four measurement planes are mounted in one chassis box and powered by a single power supply. One switch is triggered at a time by TTL pulses from the timing system.

The RHIC acceleration system operates at harmonic number 360. Speed limitations of the injection kicker require that at least two empty buckets be between adjacent bunches. Therefore bunches cannot be closer together than about 110ns. The FET switch which fires the tune kicker gives a pulse length of 140ns which will be centered on the measured bunch, fig. 3. Also shown are the coupled pulses from both the $180^{\circ}$ line and one of the $90^{\circ}$ lines. This shows that there is a flat region of about 70ns where the beam will be affected only by the pulsed line. 


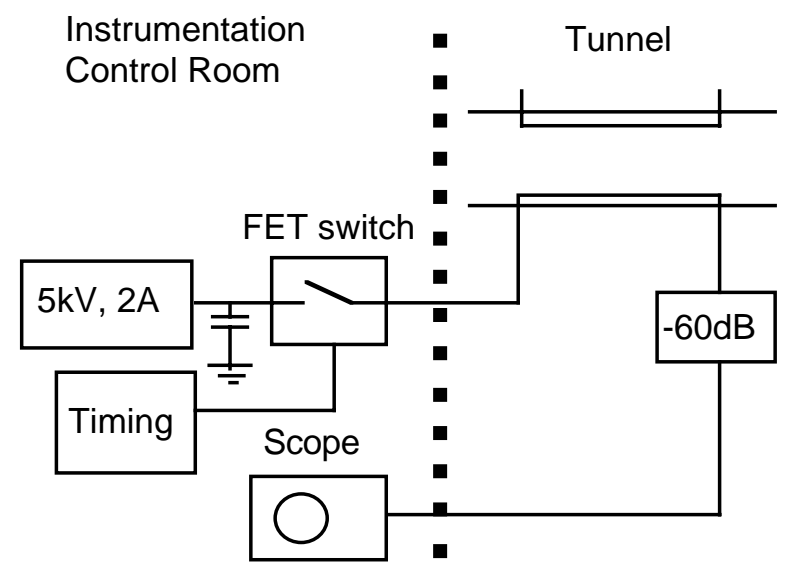

Figure 2. Electrical block diagram of kicker.

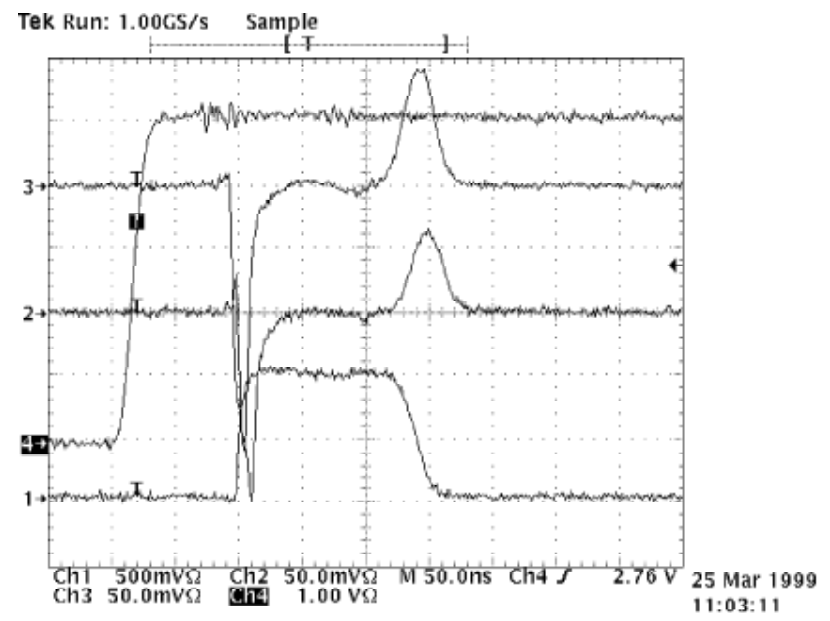

Figure 3. Oscilloscope display of trigger signal (4), output pulse (1), and coupled pulses from the $180^{\circ}$ line and one $90^{\circ}$ line $(2,3)$. The trigger delay here is $80 \mathrm{~ns}$ and the pulse is about 130ns long. A $1 \mathrm{kV}$ pulse returns as a $1 \mathrm{~V}$ pulse after $-60 \mathrm{~dB}$ of attenuation.

A ring has 161 BPM's for each plane. These are $23 \mathrm{~cm}$-long $50 \Omega$ striplines with one end shorted and the other end connected to high-speed, peak-sampling integrated processing electronics. The system is described in ref. 4. In principle any of the BPM's can be used for the measurement and one is chosen where the $\beta$-function is large.

A single kick of a gold beam at injection will cause a deflection of $11 \mu \mathrm{rad}$ giving a betatron amplitude of $780 \mu \mathrm{m}$ at the BPM we plan to use. The expected BPM noise floor for single bunch measurements $50 \mu \mathrm{m}$. Also there is a movable dual-plane BPM located near each kicker. These can be positioned to null out the closed orbit signal therefore possibly increasing the resolution to around $1 \mu \mathrm{m}$.

\section{TRIGGERING}

The FET switch is triggered by a TTL pulse from a modified BPM module. This module receives and decodes the beam synchronous event link, fig. 4. This is a timing and event channel which delivers both a revolution tick synchronous with the beam and encoded event triggers.

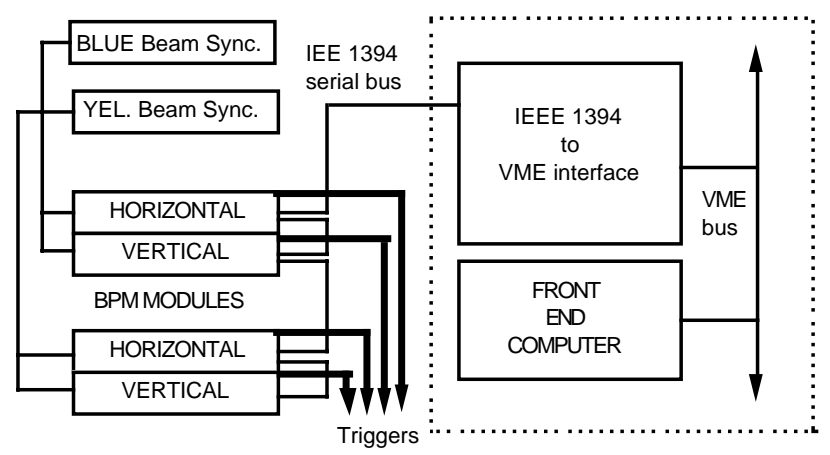

Figure 4. Block diagram of kicker timing. This is same as BPM data acquisition.

In the case of the kicker, the 'turn-by-turn data acquisition of the BPM' event will trigger a sequence of one or more kicks. The kicker trigger is supplied by the enable gate output which normally triggers data acquisition by the BPM. Each BPM and the kicker module has the appropriate delay so, on turn-by-turn acquisition, the same bunch will be kicked and observed on all BPM's. For most tune measurements the data from a single BPM per plane in each ring will be used.

All of the BPM's are connected to VME via IEEE 1394 serial bus interface. In the case of the kicker the connection will be used to read a time-stamped history of kicks for correlation to the time-stamped position measurements. Also kick delay will be adjusted over this bus.

\section{SIMULATION RESULTS}

In early operation of RHIC the tune will be measured by kicking a single bunch with one or more kicks to build up a betatron oscillation. The transverse position of this bunch will be measured on consecutive turns with one dedicated BPM at a location with a high beta function. Also being considered is white noise excitation of the beam by applying random kicks to the bunch duringthe entire measurement process and continuous low-level excitation via a phase-lock loop.

A Monte Carlo simulation was done to estimate the possible measurement accuracy and to test the performace of application software. A sinewave multiplied by a 
calculated Gaussian-shaped envelope [5] is analyzed by performing a fast Fourier Transformation (FFT) on the position data for 1024 turns. In the simulation a coupling of $90 \%$ between horizontal and vertical betatron motion has been assumed. The fractional tunes with a tune spread of 0.001 each were set to be 0.01 apart. White noise with various rms amplitudes was added to simulate the anticipated noise floor of the BPM. Noise filter algorithms are implemented in the application software to enhance fit results.

Figure 5 shows a smoothed FFT spectrum with simulated data at a signal-to-background ratio of 0.7 . The solid lines correspond to the fitted horizontal and vertical tune signals. The FFT, noise filtering and fitting were performed on a set of input signals $\left(\mathrm{q}_{\text {inp }}\right.$ ) superimposed by white noise at signal-to-background ratios from 0.1 to 1.0 .

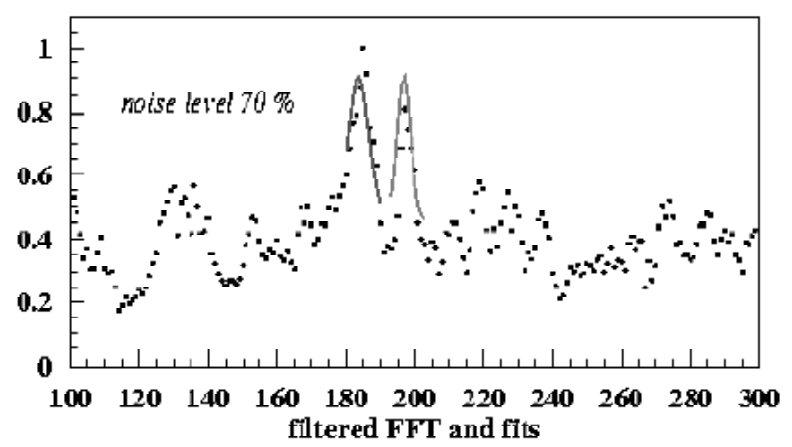

Figure 5. Smoothed FFT spectrum with simulated data with a signal-to-noise ratio of 0.7 .

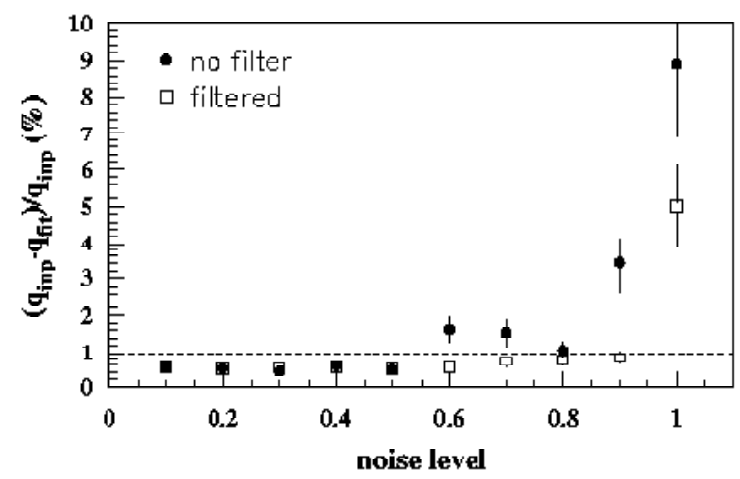

Figure 6. Measurement error ( $\Delta \mathrm{q} / \mathrm{q})$ plotted as a function of noise amplitude for filtered and unfiltered data.

Figure 6 shows the deviation of the fitted tune $\left(q_{\mathrm{fit}}\right)$ from the input value as a function of noise level for both filtered and unfiltered FFT spectra. It can be seen that while using a noise filter the average deviation from the real value stays below $1 \%$ up to a noise level of $90 \%$.
This value corresponds to an accuracy of the order of $10^{-3}$ which is required for the tune measurement. For the anticipated BPM noise level at RHIC start up, i.e. 50\%, we expect a measurement accuracy of $1 \times 10^{-3}$.

\section{DISCUSSION}

The RHIC tune system has been described. Two kicker sections in each ring will be connected in series at first and transverse kicks will be delivered to the beam via fast FET switches. For early operation tune will be measured by giving a single bunch one or more kicks to build up a betatron oscillation and following this bunch with a BPM in the turn-by-turn mode. Simulations indicate that fractional tune can be measured with an uncertainty of $<0.5 \%$.

\section{ACKNOWLEDGMENTS}

We thank Phil Cerniglia for coordinating the cable installation. Also several of the authors had very helpful discussions with Hermann Schmickler. This work was done under the auspices of the U.S. Department of Energy.

\section{REFERENCES}

1. http://www.rhichome.bnl.gov/RHIC/index.html

2. P. Cameron, R. Connolly, A. Drees, T. Ryan, H. Schmickler, T. Shea, D. Trbojevic, "ARTUS: A Rhic TUne monitor System," http://www.rhichome.bnl.gov/ AP/ap_notes/index.html

3. Behlke Electronic GmbH, http://www.eurotek.com/

4. T. Shea, P. Cameron, P. Cerniglia, J. Cupolo, C. Degen, J. Mead, R. Olsen, W. Ryan, T. Satogata, and R. Sikora, "The RHIC Position Monitor System," these proceedings.

5. R. Connolly, "Decoherence of Betatron Oscillations in RHIC," AD/RHIC/RD-118, Brookhaven National Lab, Upton, NY 11973. 